verantwortlichen chargengleichen Präparateprobe in Mehrfachansätzen unter optimierten Zellkulturbedingungen inkubiert. Zur Verhinderung unspezifischer Aktivierungen wurde den Testansätzen Interferon-alpha $(125 \mathrm{IU} / \mathrm{ml})$ zugesetzt (v. Baehr et al. J Immunol Methods 2001, 251: 63-71). Die spezifische zelluläre Aktivierung wurde am 6. Tag an Hand der zellulären Einbaurate von $\mathrm{H}^{3}$-Thymidin analysiert. 56 gesunde Kontrollprobanden wurden parallel untersucht.

Ergebnisse: Bei 17 der 21 Patienten konnte zum Untersuchungszeitpunkt die Sensibilisierung mit dem LTT nachgewiesen werden. Die maximalen Stimulationsindizes zeigten Werte zwischen 5,1 und 28,4 (MW 9,8) im Vergleich zur Kontrollgruppe (0,9-2,2, MW 1,4). Die zelluläre Reaktion war individuell dosisabhängig, d. h. das In-vitro-Reaktionsoptimum hinsichtlich der eingesetzten Verdünnungsstufen variierte zwischen den Patienten.

Schlussfolgerungen: Der LTT ist in einer optimierten, standardisierten Form geeignet zur Bestätigung allergischer Sensibilisierungen auf Penicillinpräparate. Der direkte Einsatz potenziell verantwortlicher Präparate im Test verlangt eine standardisierte Probenaufarbeitung, eine breit angelegten Dosisreihe und die parallelen Testung von Kontrollprobanden. Bei richtiger Durchführung und Interpretation der Ergebnisse kann der LTT ein kostengünstiges und effektives Verfahren zum Nachweis zellulärer Sensibilisierungen gegenüber Antibiotikaproben darstellen.

\section{Einfluss von Montelukast auf die inhalative bronchiale Allergenprovokation}

F.-W. Riffelmann, G. Droste, H. Lauter, D. Köhler

Fachkrankenhaus Kloster Grafschaft, Zentrum für Pneumologie, Beatmung und Schlafmedizin, Allergologie

Hintergrund: Leukotriene besitzen als Mediatoren bei Asthma bronchiale eine starke bronchokonstriktorische und proinflammatorische Potenz. An 16 Probanden mit Asthma bronchiale wurde in zwei Gruppen (randomisiert, cross-over) untersucht, ob eine Vormedikation mit dem Leukotrienantagonisten Montelukast (21 Tage $10 \mathrm{mg} / \mathrm{d}$ ) einen relevanten Einfluß auf die inhalative bronchiale Allergenprovokation hat und ob eine Pausierung der Medikamenteneinnahme von einem oder drei Tagen vor Provokation ausreicht.

Methode: Die Allergeninhalation erfolgte durch Verneblung handelsüblicher Provokationslösungen in der Verdünnung 1:1000, 1:100, 1:50, 1:10 und 1:5 mit einem Düsenvernebler (Fa. Pari). Alle 20 Minuten wurde die Allergendosis gesteigert, sofern die Positivkriterien (Abfall des FEV1 um 20\%, Verdopplung der oszillatorischen Resistance) noch nicht erreicht waren. Die Provokationstests wurden an Tag 1, 21, 42 und 63 durchgeführt, wobei vor der letzten Provokation eine 1- bzw. 3-tägige Einnahmepause lag.

Ergebnisse: Bei 12 von 16 Probanden zeigte sich ein unterschiedlich stark ausgeprägter allergenprotektiver Effekt (Steigerung der PD20Allergen zwischen 1,6- und 21-fach) bei Provokation mit Pollen und Milbenallergen. Im Mittelwert aller 16 Patienten ergab sich eine hochsignifikante Zunahme
( $\mathrm{p}=0,0005)$ um das 4,3-fache. Das Ausmaß der Allergenprotektion ist im Einzelfall nicht vorauszusehen und unabhängig vom Allergen oder der PD20Allergen ohne Montelukast. Es zeigt sich aber ein signifikanter Zusammenhang $(\mathrm{p}=0,0016)$ zwischen der unspezifischen bronchialen Hyperreagibilität (Histamin) und der PD20Allergen. Der allergenprotektive Effekt von Montelukast nimmt mit steigender unspezifischer Hyperreagibilität ab.

Nach 1 Tag Einnahmepause nimmt die PD20Allergen um $40 \%$, nach 3 Tagen um $68 \%$ ab.

Schlussfolgerung: Wir empfehlen, die Medikation mit Montelukast vor der Allergenprovokation abzusetzen, da im Einzelfall nicht vorauszusehen ist, ob und in welchem Ausmaß Montelukast die Sofortreaktion nach inhalativer bronchialer Allergenprovokation unterdrückt.

Die Einnahme sollte mindestens 3 Tage vor der Provokation pausiert werden.

\section{Die Anwendung einer Kurzzeit-Immuntherapie mit Pollenallergoiden und dem Adjuvans $\mathrm{MPL}^{\circledR}$ in einer dermatologischen Praxis}

G. Fiedler

Weißenfels

Hintergrund: Die Kurzzeit-Immuntherapie gewinnt als Alternative zur konventionellen Langzeittherapie zunehmend an Bedeutung. Unter Praxisbedingungen ist eine reduzierte Anzahl an Injektionen erstrebenswert und kann die Compliance der Patienten verbessern.

In Deutschland steht seit 1999 eine Kurzzeit-Immuntherapie mit nur vier Injektionen zur Verfügung. Es handelt sich um ein Präparat mit L-Tyrosin-adsorbierten Allergoiden und dem Adjuvans MPL ${ }^{\circledR}$ (POLLINEX Quattro). Die Wirksamkeit und Verträglichkeit dieser Therapie wurde in plazebokontrollierten Doppelblindstudien bei Pollenallergikern gezeigt. In dieser Studie sollte die Anwendung dieser Kurzzeit-Immuntherapie mit nur vier Injektionen in einer dermatologischen Praxis beurteilt werden.

Patienten: 89 Pollenallergiker mit Rhinokonjunktivitis und/oder Asthma wurden präsaisonal mit POLLINEX Quattro therapiert. Die Patienten erhielten vier Injektionen in wöchentlichen Abständen (300, 800, $2 \times 2000 \mathrm{SU}+50 \mu \mathrm{g}$ $\mathrm{MPL}^{\circledR} /$ Injektion).

Material und Methoden: Die Wirksamkeit wurde anhand der Verbesserung der Symptomatik und des Verbrauches an antiallergischer Medikation beurteilt. Zur Dokumentation der Verträglichkeit wurden lokale und systemische Reaktionen erfasst. Zusätzlich wurden die Werte für das spez. IgE während des Pollenfluges bestimmt.

Ergebnisse: Eine starke Besserung der Symptome stellte sich bei $61 \%$ der Patienten (1. Therapiejahr) bzw. 71\% der Patienten (2. Therapiejahr) ein. Der Verbrauch an antiallergischer Medikation reduzierte sich signifikant im Vergleich zur vorhergehenden Pollensaison. Die Werte des spez. IgE (Gräser) während des Pollenfluges verbesserten sich nach der ersten Therapie im Vergleich zum Vorjahr. 